research is to prove with evidence that through evidence itself is the most effective, secure and ethical way of practicing medicine. Even though the outlook is not encouraging, showing the importance of EBM must be the first step to change the empirical paradigm, where a doubt is perceived as a weakness and not as an opportunity. Online mentorship might be a practical solution to close the gap between poor developed and well-developed EBM environments. A personal experienced mentor could encourage and guide a young researcher to improve research quality and integrity. Asking the right questions, declaring conflicts of interests, statistical significance and reporting bias should be part of a checklist that could be revised together online. At the Universidad Nacional Autónoma de Honduras (UNAH) this could be an effective strategy. This collaboration might expand the EBM frontiers to places that finally have recognized research as an urgent need.

\section{FACTORS AFFECTING THE IMPLEMENTATION OF CARDIOVASCULAR RISK SCORING IN PRIMARY CARE; A MIXED-METHOD SYSTEMATIC REVIEW}

${ }^{1}$ Tonny Brian Muthee, 2Derick Kimathi, ${ }^{1}$ Georgia C Richards, ${ }^{3} \mathrm{Nia}$ Roberts, ${ }^{1}$ Veronika Williams, 'David Nunan, ${ }^{1}$ Carl Heneghan. ${ }^{1}$ Nuffield Department of Primary Care Health Sciences, University of Oxford https://www.phc.ox.ac.uk, Oxford, UK; ${ }^{2} K E M R I-$ Wellcome Trust Research Programme (KWTRP), Kilifi, Kenya; ${ }^{3}$ Bodleian Libraries, University of Oxford, Oxford, UK

10.1136/bmjebm-2019-EBMLive.87

Objectives This review seeks to synthesise current knowledge on factors that influence the implementation of cardiovascular risk scoring in various primary care settings as reported in primary studies.

Primary objective To explore the facilitators and barriers to the implementation of cardiovascular risk scoring in primary care.

Secondary objective To examine these factors within different contexts such as a country's Gross National Income classification and the users of the intervention.

Method We searched bibliographic databases and grey literature such as EMBASE, MEDLINE, The Cochrane Library, CINAHL, PsycINFO, Global Health and Web of Science for studies of any design relating to the topic. Titles, abstracts, and full texts were independently assessed for eligibility by two reviewers. This was followed by quality assessment and data extraction. The analysis was done through an integrated and best fit framework synthesis approach. Quantitative and qualitative forms of data were combined into a single mixed methods synthesis. The Consolidated Framework for Implementation Research was used as the guiding tool and template for this analysis. The aggregated data was coded using NVivo 12. Additional analysis was conducted to identify the facilitators and barriers common in high-income countries versus those in low and middle-income countries, those that are patient-related versus those that are healthcare provider-related and those that related to physicians versus those related to non-physicians.

Results Twenty-five studies of various designs were included in this review. Most of these studies were conducted in highincome countries. All studies included healthcare professionals as participants and reported on a total of 11 cardiovascular risk scores. The a priori framework (CFIR) used in this review was appropriate in that the extracted data fit the framework's constructs. However, one new theme emerged from the data - knowledge and belief about disease and risk. This was classified under the characteristics of individuals' domain. The factors influencing the implementation of cardiovascular risk scoring were broadly conceptualised into factors relating to the cardiovascular risk tool, users, clinical setting and healthcare system. Despite the dearth in research in low and middle-income countries, there were many similarities in the findings across all Gross National Income indices and users.

Conclusions The findings from this review show that factors influencing the implementation of cardiovascular risk scoring can be broadly conceptualised into: the healthcare system and clinical settings, the users of cardiovascular risk scoring, and the tools used for cardiovascular risk scoring. Whilst these findings reinforce the understanding that implementation processes are multifaceted and that they involve many components which act synergistically, there is limited research in the context of low and middle-income countries. Notwithstanding the need to direct resources in bridging this gap, it is also crucial that these efforts are in concert with providing high quality evidence on the clinical effectiveness of using cardiovascular risk scoring to improve cardiovascular disease outcomes of mortality and morbidity.Research in the form of effectivenessimplementation hybrid designs which will combine the elements of clinical effectiveness and implementation research to enhance public health impact is crucial.

\section{THE BARRIER OF USING DIGITAL HEALTH IN OLDER PEOPLE: A STUDY IN RURAL COMMUNITY, THAILAND}

Korravarn Yodmai. Faculty of Public Health, Mahidol University, Bangkok, Thailand

\subsection{6/bmjebm-2019-EBMLive.88}

Objectives This qualitative study aims to explore the perception of family members on using telemedicine in a rural community, Khon Kean Province, Thailand.

Method Fifty-five pairs of older people and family caregivers enrolled. This study used the structural interview for collecting data. Data analysis used the contents analysis.

Results The finding indicated that the majority of older people and their family members prefers to visit their physician in a health facility due to believe and trust the health professional skill rather than health volunteers or family members. The barrier of using telemedicine in the rural community was literacy and believe of both older people and family caregivers. Most of the older people family used the internet and have smart devices. Few older people are able to use a smart device. Older people fear to use smart devices related to a complex application and their literacy. Moreover, health conditions such as visual impairment, hearing impairment, memory impairment distributed as a barrier to learning a new technology.

Conclusions The conclusion that this era has two barriers to applying digital health in the rural community includes literacy of older people and family caregivers and self-esteem on home-health care. Developing health care technology must simple, appropriated to low literacy user, specific older adult. Using digital technology in healthcare services may cause of social inequality for the poor property, policy marker should considerate. 